\title{
Thrombocytosis in Myelodysplastic Syndromes: Not an Innocent Bystander
}

Jennifer Zikria', Naomi Galili', Wei Yann Tsai ${ }^{2}$, Huichun Zhan ${ }^{1}$, Xiaomei Ma ${ }^{3}$ and Azra Raza ${ }^{\text {* }}$

${ }^{1}$ Myelodysplastic Center, Columbia University Medical Center, New York, NY

${ }^{2}$ Mailman School of Public Health of Columbia University, New York, NY

${ }^{3}$ Yale School of Medicine, New Haven, CT

\begin{abstract}
Background: Myelodysplastic Syndromes (MDS) are typically characterized by peripheral blood cytopenias. However, some MDS is associated with thrombocytosis rather than thrombocytopenia. The prognostic significance of thrombocytosis is unknown. The purpose of this study is to determine if thrombocytosis predicts Overall Survival (OS).

Methods: We reviewed a prospective cohort of patients with myeloid disorders from 1994 to July 2011. The median duration of observation was 6.3 years. The cohort included 5,886 bone marrow biopsies or aspirates from 2,042 patients. OS of patients with thrombocytosis was compared to WHO case matched patients with normal $(100-450,000)$ and low platelet counts $(<100,000)$. All-cause mortality was evaluated using Cox proportional hazard regression modeling and Kaplan Meier methods.

Results: Thrombocytosis occurred in $5 \%(102 / 2,042)$ of patients throughout their disease. Platelet counts remained stable in $86 \%$ of patients $(1,593 / 1,856)$ and thrombocytosis was constant in $93 \%$ of patients $(86 / 92)$. Patients with thrombocytosis had significantly decreased OS compared to those with normal platelet counts in multivariate analysis adjusted for age and IPSS score $(P<0.035)$. Patients with normal platelet counts had the best prognosis and patients whose platelet counts changed during their disease course had the worst survival $(p<0.0009)$.
\end{abstract}

Conclusions: MDS patients with thrombocytosis have significantly decreased OS compared to those with normal platelet counts. The IPSS score does not accurately predict their prognosis.

Keywords: Myelodysplastic syndromes; Thrombocytosis; Prognosis; Myelodysplastic/ Myeloproliferative overlap syndromes; IPSS; Platelet count

\section{Background}

Myelodysplastic syndrome is a heterogeneous group of clonal hematopoietic stem cell diseases clinically characterized by variable cytopenia. Multiple studies have demonstrated that thrombocytopenia at diagnosis is associated with worse Overall Survival (OS) [1-5]. However, certain MDS subsets present with thrombocytosis rather than cytopenia. MDS subtypes associated with thrombocytosis include: 1) Deletion $5 q 2$ ) Refractory anemia with ringed sideroblasts and thrombocytosis (RARS-T) and 3) 3q21.q26 syndrome that affects the thrombopoietin gene [6].

The clinical significance of MDS that presents with thrombocytosis is unknown. Thrombocytosis is rare with an $8 \%$ incidence [7] in the largest previously reported case series that included 31 patients with MDS and platelets $>400,000$. Thrombocytosis in this series was associated with decreased incidence of high grade MDS, equal Acute Myelogenous Leukemia (AML) transformation, with a trend toward longer Overall Survival (OS) compared to patients with normal platelets with similar IPSS and age. In contrast, others found a decrease in OS using a series of 22 patients with platelets $>600,000$ [8]. The diagnostic criteria defining thrombocytosis has recently changed. The World Health Organization (WHO) 2008 classification defined thrombocytosis in RARS-T as having a platelet count $\geq 450,000$ in 2008 , decreased from $\geq 600,000$ in its 2003 classification. This decrease in platelet count was consistent with Essential Thrombocytosis (ET) requiring $\geq 450,000$ platelet count. Since the International Prognostic Scoring System (IPSS) includes the number of cytopenias in its scoring system, it may be inaccurate in predicting OS in thrombocytosis.
The purpose of this study is to define the incidence of thrombocytosis in MDS and determine its prognostic impact on overall survival.

\section{Methods}

We analyzed a prospective cohort of patients with myeloid disorders treated at the University of Massachusetts Medical Center (MC) in Worcester, MA; Rush University MC in Chicago, IL; St. Vincent's MC in New York, NY; and Columbia University MC in New York, NY from 1994 to July 2011 . The registry included 5,886 bone marrow biopsies and aspirates from 2,042 patients with a median age of 68 years (range 14-106 years). The median duration of follow-up was 6.3 years. We searched for patients with thrombocytosis defined as having platelet counts $>450,000$. For patients with multiple samples, any entry with platelets $\geq 450,000$ was included (see Figure 1) and subset analysis performed on patients with consistently elevated and unstable counts. Patients with Chronic Myelogenous Leukemia (CML) were excluded. Informed consent was obtained when patient's bone marrow samples were donated to the tissue repository.

*Corresponding author: Azra Raza, Director of Myelodysplastic Syndromes Center, Columbia University Medical Center, Milstein Hospital Building, 6N-435, 177 Fort Washington Avenue, New York, NY, 10032, Tel: 212-305-8291; Fax: 212-3056891; E-mail: azra.raza@columbia.edu

Received February 13, 2012; Accepted March 02, 2012; Published March 07, 2012

Citation: Zikria J, Galili N, Tsai WY, Zhan H, Ma X (2012) Thrombocytosis in Myelodysplastic Syndromes: Not an Innocent Bystander. J Blood Disord Transfus S3:002. doi:10.4172/2155-9864.S3-002

Copyright: ( 2012 Zikria J, et al. This is an open-access article distributed under the terms of the Creative Commons Attribution License, which permits unrestricted use, distribution, and reproduction in any medium, provided the original author and source are credited. 
Citation: Zikria J, Galili N, Tsai WY, Zhan H, Ma X (2012) Thrombocytosis in Myelodysplastic Syndromes: Not an Innocent Bystander. J Blood Disord Transfus S3:002. doi:10.4172/2155-9864.S3-002

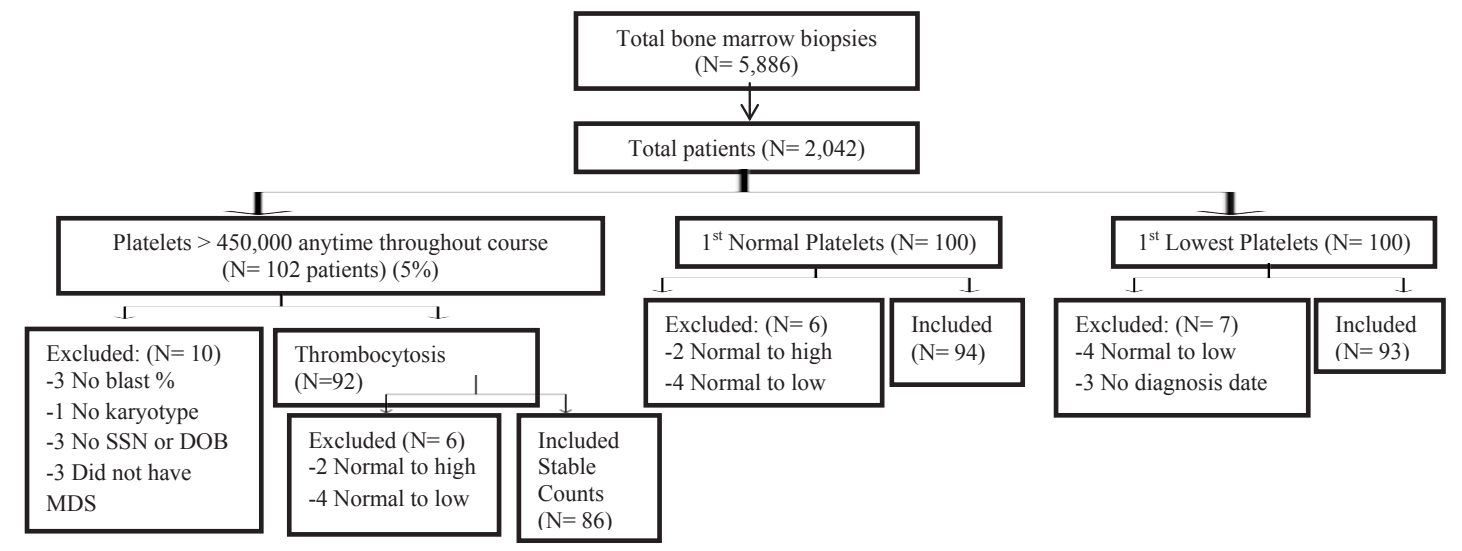

Figure 1: Selection Criteria.

\begin{tabular}{|c|c|c|c|c|}
\hline Platelet Category, Count (x10\%/L), Total (N) & Low $<100,94$ & Normal $100-450,93$ & High > 450, 86 & Univariate P score \\
\hline Age, y, median (range) & $66(14-88)$ & $68(23-92)$ & $66(36-85)$ & $<0.001$ \\
\hline Female/Male, $\%$ & $30 / 70$ & $43 / 57$ & $43 / 57$ & 0.15 \\
\hline Hemoglobin, g/dL, median, (range) & $9.4(4.2-13.6)$ & $9.6(6.2-14.8)$ & $9.2(5.2-15.1)$ & 0.005 \\
\hline Neutrophil count x109/L (range) & $1.1(0.0-10.1)$ & $1.8(0.2-32.9)$ & $3.6(0.3-37)$ & 0.14 \\
\hline Platelet count x109/L (range) & $21(1-86)$ & $239(201-334)$ & $590(450-1655)$ & 0.06 \\
\hline \multicolumn{5}{|l|}{ FAB/WHO, N, (\%) } \\
\hline RA & $42(45)$ & $34(37)$ & $20(23)$ & 1.0 \\
\hline RARS & $21(22)$ & $27(29)$ & $21(24)$ & 0.5 \\
\hline RAEB & $11(11)$ & $10(11)$ & $10(12)$ & $<0.001$ \\
\hline RAEB-t & $4(4)$ & $4(4)$ & $2(2)$ & $<0.009$ \\
\hline AML & $3(3)$ & $3(3)$ & $3(4)$ & 0.008 \\
\hline CMML & $3(3)$ & $2(2)$ & $3(4)$ & 0.004 \\
\hline Overlap MPN/MDS & $0(0)$ & $0(0)$ & $9(11)$ & 0.5 \\
\hline Systemic Mastocytosis & $0(0)$ & $0(0)$ & $1(1)$ & $\mathrm{N} / \mathrm{A}$ \\
\hline RCMD & $0(0)$ & $0(0)$ & $2(2)$ & 0.2 \\
\hline Other & $5(5)$ & $6(6)$ & $8(9)$ & 0.9 \\
\hline N/A & $5(5)$ & $7(8)$ & $7(8)$ & 0.9 \\
\hline \multicolumn{5}{|l|}{ CYTOGENETICS, $\mathrm{N},(\%)^{1}$} \\
\hline Good & $46(49)$ & $74(80)$ & $63(73)$ & 1.0 \\
\hline Intermediate & $21(22)$ & $12(13)$ & $13(15)$ & 0.2 \\
\hline Poor & $27(29)$ & $7(7)$ & $10(12)$ & 0.001 \\
\hline \multicolumn{5}{|l|}{ IPSS SUBTYPE, N, (\%) } \\
\hline Low & $5(5)$ & $46(49)$ & $41(48)$ & 1.0 \\
\hline Intermediate-1 & $57(61)$ & $34(37)$ & $35(41)$ & $<0.001$ \\
\hline Intermediate-2 & $22(23)$ & $9(10)$ & $7(8)$ & $<0.001$ \\
\hline High & $10(11)$ & $4(4)$ & $3(3)$ & $<0.001$ \\
\hline \multicolumn{5}{|l|}{ RACE, N, (\%) } \\
\hline Asian & $1(1)$ & $2(2)$ & $2(2)$ & 0.2 \\
\hline Black & $4(5)$ & $3(3)$ & $4(5)$ & 0.5 \\
\hline Hispanic & $0(0)$ & $2(2)$ & $1(1)$ & 0.3 \\
\hline White & $83(88)$ & $78(84)$ & $71(83)$ & 1.0 \\
\hline Other & $0(0)$ & $1(1)$ & $2(2)$ & 0.4 \\
\hline Unknown & $6(6)$ & $7(8)$ & $6(7)$ & 0.9 \\
\hline \multicolumn{5}{|l|}{ SURVIVAL STATUS } \\
\hline Not Alive & $84(89)$ & $71(76)$ & $60(70)$ & \\
\hline Alive & $10(11)$ & $22(24)$ & $26(30)$ & \\
\hline
\end{tabular}

${ }^{1}$ See Supplemental Table $1 \mathrm{~A}$ for specific cytogenetic abnormalities

Table 1: Baseline Characteristics of Patients with Myeloid Malignancies 


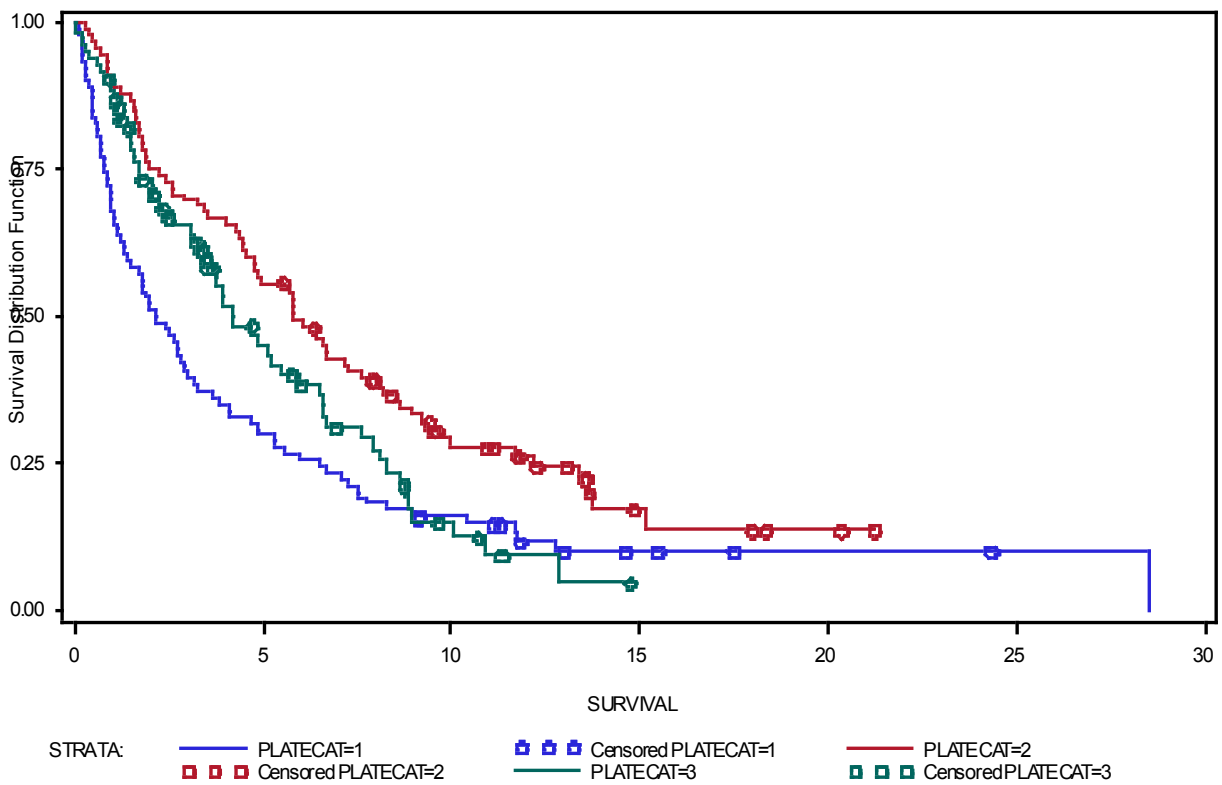

Red- Normal Platelets (100,000-450,000); Blue- Low Platelets (<100,000); Green- High Platelets ( $>450,000)$. X-axis Survival (years). Y-axis Percentage

Figure 2: Kaplan Meier Survival Curves based on Platelet Count.

Patients were stratified according to: WHO Classification, IPSS classifications and cytogenetics. Karyotype classification utilized the IPSS cytogenetic scoring into good (normal, deletion 5q alone, or deletion Y alone), poor (chromosome 7 abnormality or complex karyotype [ $>3$ different cytogenetic abnormalities]) and intermediate (all other aberrations, including addition of Chromosome 1 or Chromosome 8) [9]. Overall survival (OS) was calculated from the time of first sample collection to time of death from any cause. If the date of diagnosis was unknown, the date of first available bone marrow was used as the date of diagnosis.

Control groups were matched for WHO subtype for normal platelet count $(100-450,000)$ and low platelet count $(<100,000)$. After sorting the database by platelet count, the normal platelet group was selected from the first consecutive 50 patients with platelet counts above and below the median count of 275,000 . The low control group was selected from the first consecutive 100 patients with the lowest platelet count. Since MyeloProliferative Neoplasms (MPN) do not occur by definition in normal or low platelet counts, RA was substituted in these groups.

\section{Statistical Analysis}

Patient characteristics were compared using the Fishers exact test for categorical data. Patients last known to be alive were statistically censored. OS was evaluated using unadjusted and adjusted Cox proportional hazard regression modeling. Multivariate analysis for OS was adjusted for IPSS risk group at first sample collection and ageadjusted mortality. OS curves were constructed using Kaplan-Meier methods. Subset analysis weighed WHO subtypes (RA and MPN) that were not equally represented in the platelet categories.

\section{Results}

Platelet count remained stable throughout patients' disease course regardless of platelet category. Out of the 5,886 bone marrow biopsies from 2,042 patients, 1,856 were included in analysis and 186 patients were excluded due to missing platelet counts (Figure 1). Most MDS patients regardless of platelet count at presentation had stable platelets from presentation to death $(86 \%, 1,593 / 1,856)$. Out of the $14 \%$ $(263 / 1,856)$ of patients whose platelet counts changed, most transitioned from normal to thrombocytopenia $(9 \%, 170 / 1,856)$ likely representing treatment side effects or disease progression. Only $2 \%(45 / 1,856)$ with either thrombocytopenia or $<1 \%(15 / 1,856)$ thrombocytosis at presentation subsequently developed normal platelet count. Less than $1 \%$ of MDS cases changed from thrombocytopenia to thrombocytosis $(13 / 1,856)$, from normal counts to thrombocytosis $(19 / 1,856)$, or from thrombocytosis to thrombocytopenia $(1 / 1,856)$ (Figure 1A).

The incidence of thrombocytosis occurring at anytime during disease course was $5 \%(102 / 1,856)$. Out of the 102 patients with thrombocytosis, 86 (93\%) with consistently increased counts were included in analysis, 10 were excluded due to missing data or having CML and 6 (7\%) with unstable thrombocytosis were excluded. However, subset analysis was performed comparing patients with consistently elevated counts to those with unstable thrombocytosis.

Control groups were matched for WHO subtype for normal platelet count and low platelet count. In the normal platelet category, out of the 100 selected, 94 were analyzed and 6 were excluded due to unstable platelet counts ${ }^{\mathrm{i}}$. In the low platelet control group, out of the 100 selected, 93 patients were included in analysis and 7 were excluded ( 3 due to missing data and 4 with unstable platelet counts).

Age, gender, hemoglobin count and race were similar in the 3 groups (see Table 1 for Baseline Characteristics). The median platelet count was 21,000 in the low platelet, 239,000 in the normal and 590,000

iTwo patients initially presented with normal platelet counts and subsequently developed thrombocytosis, and four initially presented with normal platelet counts but later became thrombocytopenic 


\begin{tabular}{|c|c|c|c|c|}
\hline Characteristic, $\mathbf{N}$ & OS, y, median, (range) & OS, Hazard Ratio $(95 \% \mathrm{Cl})^{*}$ & Univariate $P$ Value & Multivariate P Value \\
\hline $\begin{array}{l}\text { Age }(\mathrm{y}), \mathrm{N}, \text { median, range } \\
\leq 60, \mathrm{~N}=77,52(14-60) \\
60-70, \mathrm{~N}=91,66(61-69) \\
\geq 70, \mathrm{~N}=105,75(70-92)\end{array}$ & $\begin{array}{c}5.3(0-28.5) \\
3.1(0.1-18.3) \\
3.2(0.1-13.7)\end{array}$ & $\begin{array}{l}1.0 \\
1.9 \\
2.2\end{array}$ & $\begin{array}{c}\mathrm{N} / \mathrm{A} \\
0.001 \\
<0.001\end{array}$ & $\begin{array}{r}0.001 \\
<0.001\end{array}$ \\
\hline $\begin{array}{l}\text { IPSS } \\
\text { Low, } \mathrm{N}=92 \\
\text { Intermediate } 1, \mathrm{~N}=126 \\
\text { Intermediate } 2, \mathrm{~N}=38 \\
\text { High, } \mathrm{N}=17\end{array}$ & $\begin{array}{l}5.8(0.2-24.3) \\
3.4(0.0-28.5) \\
1.5(0.3-17.4) \\
2.3(0.1-6.6)\end{array}$ & $\begin{array}{l}1.0 \\
1.9 \\
3.8 \\
5.8\end{array}$ & $\begin{aligned} & \mathrm{N} / \mathrm{A} \\
< & 0.001 \\
< & 0.001 \\
< & 0.001\end{aligned}$ & $\begin{array}{l}\mathrm{N} / \mathrm{A} \\
0.002 \\
0.001 \\
0.001\end{array}$ \\
\hline $\begin{array}{l}\text { Platelet category (Count) } \\
\text { Low }(<100,000) \\
\text { Normal }(100,000-450,000) \\
\text { High }(>450,000)\end{array}$ & $\begin{array}{c}2.2(0.1-28.5) \\
5.8(0.3-21.2) \\
3.3(0-14.7)\end{array}$ & $\begin{array}{l}1.8 \\
1.0 \\
1.5\end{array}$ & $\begin{array}{c}0.001 \\
\mathrm{~N} / \mathrm{A} \\
0.065\end{array}$ & $\begin{array}{c}0.002 \\
\mathrm{~N} / \mathrm{A} \\
0.035\end{array}$ \\
\hline
\end{tabular}

${ }^{1} \mathrm{~N}-\mathrm{Number}$; OS-Overall survival; y-years; Cl-Confidence Interval; N/A- Not applicable

Table 2: Multivariate Analysis of Prognostic Parameters for Survival ${ }^{1}$.

in the high group. Median neutrophil count was also increased in the high platelet category with median count $3.6 \times 10^{9} / \mathrm{L}$ compared to 1.8 $\mathrm{x} 10^{9} / \mathrm{L}$ in the normal and $1.1 \times 10^{9} / \mathrm{L}$ in the low groups. The distribution for all WHO subtypes was similar in the 3 groups with the exception of MPN. Subset statistical analysis, however, found no change in OS due to unequal distribution; likely because both MPN and RA have better survival. Cytogenetics and IPSS score were similar in the high and normal platelet categories. Patients in the low platelet category had adverse cytogenetics and a higher IPSS than both the normal and high platelet groups.

Kaplan Meier survival curve based on platelet count demonstrates that thrombocytosis had a significantly worse OS compared to normal platelets (Figure 2). Thrombocytosis had a median OS of 3.3 years compared to 5.8 years in the normal platelet group. Thrombocytopenia had the lowest OS with 2.2 years. Univariate analysis for older age $(>60$ years old), lower hemoglobin level, higher neutrophil count, specific WHO subtypes (RAEB, RAEB-t and MDS evolving into AML), cytogenetics, IPSS intermediate/high risk subtypes and low platelet count at diagnosis significantly affected OS (see Table 1). Gender and race did not have a statistical affect OS. Thrombocytosis decreased OS in univariate analysis $(\mathrm{P}<0.065)$ and multivariate analysis adjusted for IPSS and age-adjusted mortality $(\mathrm{P}<0.035, \mathrm{HR} 1.5$, Table 2$)$. Decreased hemoglobin level and increased neutrophil count were also significantly prognostic of decreased OS in multivariate analysis $(\mathrm{p}<0.03$, HR 0.92$)$ and (0.001, HR 1.5), respectively.

\section{Discussion}

To date, this is the largest reported cohort of MDS patients with thrombocytosis. Surprisingly, the current analysis found that thrombocytosis is an important adverse prognostic factor that predicts for a significantly decreased OS in multivariate analysis after adjustment for age and IPSS score. In our cohort the incidence of thrombocytosis was $5 \%$. Patients with thrombocytosis regardless of IPSS or age, have a median OS of 3.3 years; which is comparable to the OS of patients with IPSS intermediate 1 (median OS 3.4 years). Perhaps patients with thrombocytosis may benefit from earlier treatment than their IPSS score indicates. However, it should be noted that no treatment has of yet been proven to prolong OS in low-grade MDS. Thus, this dataset did not examine the effect of various treatments on OS.

This study has several strengths. Unlike previous studies, the definition of thrombocytosis is in agreement with the WHO revised 2008 classification of overlap MDS/MPN, having a persistent platelet count $\geq 450,000$. In addition, patients were matched by WHO classification instead of IPSS. Since cytopenia is inherent in calculating IPSS, it may not accurately predict the prognosis of patients with thrombocytosis. Baseline characteristics (including cytogenetics and IPSS) for normal and high platelet groups were equally represented when matched by WHO classifications.

However, this study is unable to compare high- to low- platelet counts since matching patients by WHO subtypes alone results in groups with differing cytogenetics and IPSS. Since others have demonstrated that patients with platelets $\leq 30,000$ have a worse prognosis than those with $\leq 100,000$ [1]; our low platelet group is expected to have a particularly poor prognosis since $76 \%(71 / 93)$ had platelets $\leq 30,000$. Thus, additional studies comparing the prognostic impact of thrombocytosis with thrombocytopenia must be matched for WHO, IPSS and age.

Patients with an elevated platelet count that did not remain consistently elevated during their natural history underwent subset analysis. Variable platelet counts were associated with disease progression over time. Patients with unstable platelets had the shortest OS when compared to those with stable low-, high-, or normal-platelet counts. The median OS of 3.2 years (0.8- 4.5) was significantly less ( $p$ $<0.0009$, HR 5.7) compared to normal stable platelet counts. All six patients (6/92) whose platelet count changed over time had normal platelets at presentation. Two patients developed thrombocytosis and four patients later developed thrombocytopenia. Two patients had additional cytogenetic abnormalities in their repeat bone marrow biopsy.

Interestingly, platelet counts in $86 \%$ of patients in our cohort remained stable from presentation to death. Once a patient developed thrombocytosis, almost all patients (93\%) continued to exhibit thrombocytosis throughout the remainder of their disease. The stability of the platelet count suggests that it is associated with the underlying disease representing an epiphenomenon with a worse OS. It is unclear if thrombocytosis is the cause (due to complications from elevated platelet counts) or effect (due to a more aggressive underlying biology) of decreased OS.

In ET, thrombocytosis itself is associated with increased hemorrhage [10] and thrombosis in patients over 60 years of age [11]. Patients with JAK2 mutation (V617F) in MPN are especially at risk for having increased venous and arterial thrombosis [12]. Similarly, patients with thrombocytosis in MDS may be especially at risk for complications, since both MDS and thrombotic events increase with age and JAK2 mutations in overlap MDS/MPN is common. 
Alternatively, thrombocytosis in MDS represents a unique underlying disease. Indeed several mechanisms leading to thrombocytosis has recently been elucidated in all MDS subtypes characterized by elevated platelets. Depending on the platelet threshold definition, 30-90\% RARS-T harbored JAK2 mutation, with higher platelet counts associated with the mutation [13,14]. Furthermore, in RARS-T the MPL mutation results in transcriptional activation of the thrombopoietin gene located at 3q26 [13,15]. Both JAK2 and MPL lead to constitutive activation of cytokine-regulated intracellular signaling pathways and are mutually exclusive. In contrast, loss-offunction mutations involved in epigenetic regulation, such as TET2 (ten-eleven-translocation-2) and ASXL1 (additional sex-comb like 1), have also been found in MDS subtypes with thrombocytosis. TET2 converts 5-methylcystosine into 5-hydroxymethylcystosine and can occur before or after JAK2 mutation in RARS-T [16]. ASXL1 encodes a member of the polycomb family of chromatin-binding protein and its mutation occurs in $>40 \%$ CMML [17]. When a TET2 mutation is acquired in MPN after the JAK2 V617F mutation it is associated with disease progression [18]. Another mutation identified in RARS-T and in all MDS with ringed sideroblasts (RS), is SF3B1 $[19,20]$. This protein is an essential component of the spliceosome complex that joins exons from precursor RNA molecules containing both introns and exons to generate a specific messenger RNA. Presently, how the biological function of SF3B1 mutation relates to disease pathology is unclear. Additionally, several micro RNA (miR) in deletion $5 \mathrm{q}$ syndrome have been implicated in causing thrombocytosis, including miR-145, miR-146a and miR-150. In vivo inhibition of miR-146a in vivo results in increased interleukin-6 levels (IL-6) leading to increased megakaryocyte development [21]. Loss of miR145 results in increased Fli-1 [22], an ETS-family transcription factor involved in megakaryocytic and erythroid differentiation. The over-expression of miR-145 or inhibition of Fli-1, in vivo causes the reciprocal effect with decreased production of megakaryocytic cells relative to erythroid cells. Furthermore, deletion of $5 \mathrm{q}$ was associated with increased miR150 and decreased mRNA expression of MYB, a transcription factor [23] whose decreased expression promotes megakaryocytopoiesis [24]. Together, these insights into the molecular mechanisms underlying thrombocytosis in MDS support the hypothesis of its unique disease biology.

Several clinical questions remain unanswered. First, it is unknown if MDS associated with thrombocytosis exhibits increased leukemic transformation. Second, it is unclear whether current treatments for MDS or ET, including myelosuppression, hypomethylating agents, JAK2 inhibitors or platelet-lowering agents will impact OS.

In conclusion, MDS with thrombocytosis represents a subgroup of patients with a significantly decreased OS which is not accurately predicted by the IPSS. Thrombocytosis in MDS is thus not an innocent bystander. It is unknown if this poor prognosis occurs due to complications from thrombocytosis itself or due to a unique underlying biology representing an epiphenomenon. The latter seems more likely since mechanisms causing thrombocytosis have recently been elucidated in all MDS subtypes characterized by thrombocytosis.

\section{References}

1. Gonzalez-Porras JR, Cordoba I, Such E, Nomdedeu B, Vallespi T, et al. (2011) Prognostic impact of severe thrombocytopenia in low-risk myelodysplastic syndrome. Cancer 117: 5529-5537.
2. Al Ameri A, Jabbour E, Garcia-Manero G, O'Brien S, Faderl S, et al. (2011) Significance of thrombocytopenia in myelodysplastic syndromes: associations and prognostic implications. Clin Lymphoma Myeloma Leuk 11: 237-241.

3. Kantarjian H, Giles F, List A, Lyons R, Sekeres MA, et al. (2007) The incidence and impact of thrombocytopenia in myelodysplastic syndromes. Cancer 109: 1705-1714.

4. Sekeres MA, Schoonen WM, Kantarjian H, List A, Fryzek J, et al. (2008) Characteristics of US patients with myelodysplastic syndromes: results of six cross-sectional physician surveys. J Natl Cancer Inst 100: 1542-1551.

5. Kantarjian H, O'Brien S, Ravandi F, Cortes J, Shan J, et al. (2008) Proposa for a new risk model in myelodysplastic syndrome that accounts for events not considered in the original International Prognostic Scoring System. Cancer 113: $1351-1361$

6. Perez Sanchez I, Perez Corrala A, Menarguez Palanca J, Mayayo Crespo M, Escudero Soto A, et al. (2003) Sideroblastic anaemia with reactive thrombocytosis versus myelodysplastic/myeloproliferative disease. Leuk Lymphoma 44: 557-559.

7. Kodali D, Mesa H, Rawal A, Cao Q, Gupta P (2007) Thrombocytosis in myelodysplastic and myelodysplastic/myeloproliferative syndromes. Leuk Lymphoma 48: 2375-2380.

8. Cabello Al, Collado R, Ruiz MA, Martínez J, Navarro I, et al. (2005) A retrospective analysis of myelodysplastic syndromes with thrombocytosis: reclassification of the cases by WHO proposals. Leuk Res 29: 365-370.

9. Greenberg P, Cox C, LeBeau MM, Fenaux P, Morel P, et al. (1997) International scoring system for evaluating prognosis in myelodysplastic syndromes. Blood 89: $2079-2088$.

10. Fenaux P, Simon M, Caulier MT, Lai JL, Goudemand J, et al. (1990) Clinical course of essential thrombocythemia in 147 cases. Cancer 66: 549-556

11. Passamonti F, Rumi E, Arcaini L, Boveri E, Elena C, et al. (2008) Prognostic factors for thrombosis, myelofibrosis, and leukemia in essential thrombocythemia: a study of 605 patients. Haematologica 93: 1645-1651.

12. Dahabreh IJ, Zoi K, Giannouli S, Zoi C, Loukopoulos D, et al. (2009) Is JAK2 $\mathrm{V} 617 \mathrm{~F}$ mutation more than a diagnostic index? A meta-analysis of clinical outcomes in essential thrombocythemia. Leuk Res 33: 67-73.

13. Hellstrom-Lindberg E, Cazzola M (2008) The role of JAK2 mutations in RARS and other MDS. Hematology Am Soc Hematol Educ Program 52-59.

14. Malcovati L, Della Porta MG, Pietra D, Boveri E, Pellagatti A, et al. (2009) Molecular and clinical features of refractory anemia with ringed sideroblasts associated with marked thrombocytosis. Blood 114: 3538-3545

15. Schnittger S, Bacher U, Haferlach C, Dengler R, Kröber A, et al. (2008) Detection of an MPLW515 mutation in a case with features of both essential thrombocythemia and refractory anemia with ringed sideroblasts and thrombocytosis. Leukemia 22: 453-455.

16. Szpurka H, Jankowska AM, Makishima H, Bodo J, Bejanyan N, et al. (2010) Spectrum of mutations in RARS-T patients includes TET2 and ASXL1 mutations. Leuk Res 34: 969-973.

17. Bejar R, Stevenson K, Abdel-Wahab O, Galili N, Nilsson B, et al. (2011) Clinical effect of point mutations in myelodysplastic syndromes. N Engl J Med 364 2496-2506.

18. Schaub FX, Looser R, Li S, Hao-Shen H, Lehmann T, et al. (2010) Clona analysis of TET2 and JAK2 mutations suggests that TET2 can be a late event in the progression of myeloproliferative neoplasms. Blood 115: 2003-2007.

19. Malcovati L, Papaemmanuil E, Bowen DT, Boultwood J, Della Porta MG, et al. (2011) Clinical significance of SF3B1 mutations in myelodysplastic syndromes and myelodysplastic/myeloproliferative neoplasms. Blood 118: 6239-6246.

20. Yoshida K, Sanada M, Shiraishi Y, Nowak D, Nagata Y, et al. (2011) Frequent pathway mutations of splicing machinery in myelodysplasia. Nature 478: 64-69.

21. Starczynowski DT, Kuchenbauer F, Argiropoulos B, Sung S, Morin R, et al (2010) Identification of miR-145 and miR-146a as mediators of the $5 q$ syndrome phenotype. Nat Med 16: 49-58.

22. Kumar MS, Narla A, Nonami A, Mullally A, Dimitrova N, et al. (2011) Coordinate loss of a microRNA and protein-coding gene cooperate in the pathogenesis of $5 q-$ syndrome. Blood 118: 4666-4673. 
Citation: Zikria J, Galili N, Tsai WY, Zhan H, Ma X (2012) Thrombocytosis in Myelodysplastic Syndromes: Not an Innocent Bystander. J Blood Disord Transfus S3:002. doi:10.4172/2155-9864.S3-002

Page 6 of 6

23. Hussein K, Theophile K, Büsche G, Schlegelberger B, Göhring G, et al. (2010) Significant inverse correlation of microRNA-150/MYB and microRNA-222/p27 in myelodysplastic syndrome. Leuk Res 34: 328-334.
24. Emambokus N, Vegiopoulos A, Harman B, Jenkinson E, Anderson G, et al. (2003) Progression through key stages of haemopoiesis is dependent on distinct threshold levels of c-Myb. EMBO J 22: 4478-4488.

This article was originally published in a special issue, Thrombolic \& Thrombocyłopenic Purpurea handled by Editor(s). Dr. Han-Mou Tsai, Pennsylvania State University, USA. 